Editorial

\title{
Foreword to the Special Section on the Spring Conference on Computer Graphics 2015 (SCCG 2015)
}

\section{Guest Editors}

It is our pleasure to present this special section of Computers \& Graphics (C\&G), featuring the selected best papers presented at the 31st Spring Conference on Computer Graphics 2015 (www. sccg.sk), which was held April 22-24, 2015 in Smolenice, Slovakia. The venue is probably the oldest regular annual meeting of computer graphics in Central Europe, covering all relevant innovative ideas in computer graphics, image processing and their applications. The philosophy of SCCG is to bring together top experts and young researchers in CG in order to support a good and sustained communication channel for East-West European exchange of prospective ideas.

The SCCG call for papers attracted 20 submissions, 6 short papers and posters, and invited talks. Together with an international program committee, consisting of 64 experts in the topic areas, we handled a highly competitive and selective review process. Two manuscripts were selected according to the highest marks received through $C \& G$ reviewing process according to Journal criteria. These papers cover two different fields: computer animation and image representation.

M. Chládek and R. Ďurikovič approach animating shallow water surfaces through particle representation. Smoothed particle hydrodynamics is used to solve the shallow water equation by which the authors avoid discretizing the whole simulation domain to easily handle sparse and irregular simulation domains [1].

Last, but not least the work by A. Reh, A. Amirkhanov, J. Kastner, E. Gröller, and C. Heinzl presents a novel integrated visual analysis tool to evaluate series of X-ray Computational Tomography (XCT) data. A feature tracking algorithm is applied to detect changes of features throughout the series as events. As explicit tracking is not always possible, the Authors introduce the computation of a Tracking Uncertainty [2].

\section{References}

[1] Chládek Michal, Roman Durikovič. Particle-based shallow water simulation for irregular and sparse simulation domains. Comput Graph 2015;53:170-6. 〈〈http://www.sciencedirect.com/science/article/pii/S0097849315000412〉.

[2] Reh Andreas, Amirkhanov Aleksandr, Kastner Johann, Gröller Eduard, Heinzl Christoph. Fuzzy feature tracking: Visual analysis of industrial 4D-XCT data. Comput Graph 2015;53:177-84. 〈http://www.sciencedirect.com/science/arti cle/pii/S0097849315000400〉.

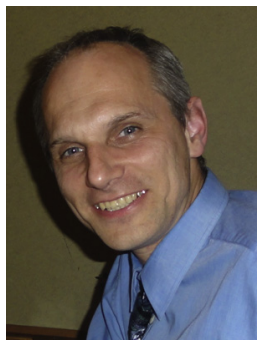

Roman Durikovič is a full professor at Comenius University in Bratislava, Slovakia, heading the Computer Graphics and Visualization Group. He was an invited professor at Tokyo University Japan, (until 2011), associated professor at Comenius University in Bratislava (until 2010), professor The University of Aizu, Japan (until 2005), invited researcher at Kyushu University, Japan (until 1999), postdoctoral JSPS fellow at Hiroshima University (until 1998). He studied Numerical Analysis and obtained his RNDr. from Comenius University in Slovakia 1998, Ph.D. in Computer Science from the Hiroshima University in Japan 1996. He spent a research visit at Groningen University, The Netherlands and University of Utrecht, The Netherlands (1991).

His research interests are in physically based animations, animation of natural phenomena, physically based rendering, GPU acceleration techniques and shape modeling. Ďurikovič co-authored the books "IT Computer Graphics" written in Japanese, "Heterogeneous Objects Modeling and Applications", "Mathematical Analysis 3" and, "Numerical Analysis for Computer Scientists", written in Slovak. He is a local organizer of SCCG conference from 2005 and paper co-chair SCCG 2011, 2014.

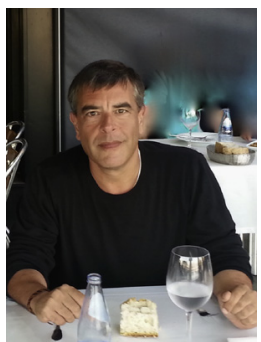

Luís Paulo Santos is a professor at the Department of Informatics, Universidade do Minho, Braga, Portugal. He was awarded his Ph.D. degree by this same university in 2001, on the area of Parallel Computing, particularly on handling uncertainty for load balancing by resorting to Bayesian networks. His research interests include Physically Based Rendering, High Performance Parallel Computer Grphics, Computer Generated Holography and Computer Vision. He has published several papers on international journals and conferences within these areas of knowledge. He acts regularly as a member of the Program Committee of several international conferences, chaired the IPC of SCCG'2015 and organized a few international conferences, such as ICEGOV'2015, VSGames 2010, VAST'2008 and EGPGV'2006. He is Associate Editor of Elsevier Computers \& Graphics, vice-president of the portuguese chapter of Eurographics and will act as short papers program co-chair for the Eurographics'2016 conference.

Roman Ďurikovič, Luís Paulo Santos E-mail address: roman.durikovic@fmph.uniba.sk (R. Ďurikovič), psantos@di.uminho.pt (L. Paulo Santos)

Received 27 May 2015; accepted 27 May 2015 